\title{
T-piece versus self-inflating bag ventilation in preterm neonates at birth
}

\begin{abstract}
Ruth Guinsburg, ${ }^{1}$ Maria Fernanda Branco de Almeida, ${ }^{1}$ Junia Sampel de Castro, ${ }^{1}$ Walusa Assad Gonçalves-Ferri, ${ }^{2}$ Patricia Franco Marques, ${ }^{3}$ Jamil Pedro Siqueira Caldas, ${ }^{4}$ Vera Lucia Jornada Krebs, ${ }^{5}$ Ligia Maria Suppo de Souza Rugolo, ${ }^{6}$ João Henrique Carvalho Leme de Almeida, ${ }^{7}$ Jorge Hecker Luz, ${ }^{8}$ Renato S Procianoy, ${ }^{9}$ José Luiz Muniz Bandeira Duarte, ${ }^{10}$ Marcia Gomes Penido, ${ }^{11}$ Daniela Marques de Lima Mota Ferreira, ${ }^{12}$ Navantino Alves Filho, ${ }^{13}$ Edna Maria de Albuquerque Diniz, ${ }^{14}$ Juliana Paula Santos, ${ }^{15}$ Ana Lucia Acquesta, ${ }^{16}$ Cristina Nunes dos Santos, ${ }^{17}$ Maria Rafaela Conde Gonzalez, ${ }^{18}$ Regina PG Vieira Cavalcanti da Silva, ${ }^{19}$ Jucile Meneses, ${ }^{20}$ José Maria de Andrade Lopes, ${ }^{21}$ Franciscó Eulógio Martinez ${ }^{2}$
\end{abstract}

For numbered affiliations see end of article.

\section{Correspondence to}

Dr Ruth Guinsburg, Division of Neonatal Medicine,

Escola Paulista de Medicina, Universidade Federal de São Paulo, São Paulo CEP 01410020,Brazil; ruthgbr@netpoint. com.br, ruth.guinsburg@ unifesp.br

Received 12 November 2016 Revised 21 April 2017 Accepted 25 April 2017 Published Online First 29 June 2017

\section{CrossMark}

To cite: Guinsburg $R$, de Almeida MFB, de Castro JS, et al. Arch Dis Child Fetal Neonatal Ed 2018;103:F49_ F54.

\section{ABSTRACT}

Objective To verify whether the use of the T-piece resuscitator compared with the self-inflating bag in preterm infants ventilated at birth modifies survival to hospital discharge without major morbidities.

Design Pragmatic prospective cohort study.

Setting 20 Brazilian university hospitals of Brazilian Network on Neonatal Research. Patients were 1962 inborn infants in 2014-2015 ventilated at birth with 23-33' weeks gestation and birth weight 400-1499 g without malformations. Patients transferred until the 27 th day after birth were excluded.

Interventions Positive pressure ventilation at birth with T-piece resuscitator or self-inflating bag without positive end expiratory pressure valve. Intervention with ventilation followed the Brazilian Society of Pediatrics guidelines. The choice of the equipment was at the neonatologist's discretion in each delivery. The main outcome measures were survival to hospital discharge without bronchopulmonary dysplasia, severe peri-intraventricular haemorrhage and periventricular leucomalacia. Logistic regression adjusted for confounding variables was applied for main outcome.

Results 1456 (74\%) were only ventilated with T-piece resuscitator and 506 (26\%) with the self-inflating bag. The characteristics of those ventilated with T-Piece resuscitator versus self-inflating bag were birth weight $969 \pm 277$ vs $941 \pm 279$ g, gestational age $28.2 \pm 2.5$ vs $27.8 \pm 2.7$ weeks and survival to hospital discharge without major morbidities $47 \%$ vs $35 \%$. Logistic regression adjusted for maternal characteristics, obstetric and neonatal morbidities showed that the T-piece resuscitator increased the chance of survival to hospital discharge without major morbidities ( $\mathrm{OR}=1.38$; 95\% Cl 1.06 to 1.80; Hosmer-Lemeshow goodness of fit: 0.695).

Conclusion This study is the first that highlights the effectiveness of T-piece resuscitator ventilation in improving relevant outcomes in preterm neonates.

\section{INTRODUCTION}

Most newborn infants with gestational age $<34$ weeks need positive pressure ventilation during cardiorespiratory transition at birth. Among 5857 very low birth weight infants (VLBW) with

\section{What is already known on this topic?}

- $60 \%-70 \%$ of very low birth weight preterm newborns (23-33 weeks' gestation) receive positive pressure ventilation during cardiorespiratory transition at birth.

- In simulators, inspiratory pressure, positive end expiratory pressure, tidal volume and inspiratory time are administered more consistently with the T-piece resuscitator compared with the selfinflating bag.

- Despite this apparent advantage regarding lung mechanics, small clinical trials in newborns do not demonstrate consistent improved clinical outcomes with the T-piece.

\section{What this study adds?}

- In preterm neonates (23-33 weeks' gestation) without malformations who received ventilation at birth, use of T-piece resuscitator was associated with increased survival without major morbidities.

gestational age of 23-33 weeks without malformations born in 2012-2015 in 20 public Brazilian university hospitals, $64 \%$ received positive pressure ventilation with facial mask or endotracheal tube in the delivery room. ${ }^{1}$ A study of the NICHD Neonatal Research Network, with data from 9565 newborns with gestational age less than 29 weeks born between 2003 and 2007, showed that 67\% received positive pressure ventilation at birth. ${ }^{2}$

The respiratory transition at birth consists of three distinct phases that overlap in the first minutes of life: in the first, the airways are full of liquid, and respiratory support aims at lung fluid clearance; in the second phase, lung fluid is still in the interstitial space and can return to the airways if the lung is not expanded; and in the last phase, the issues related to lung fluid are not as relevant, and those linked to 
gaseous exchange and uniform ventilation in an immature lung begin to have greater importance to respiratory homeostasis. ${ }^{3}$ In this context, the equipment of choice to ventilate the preterm newborn has been the subject of controversies; two devices are the most employed in the Brazilian scenario: the T-piece resuscitator and the self-inflating bag.

Studies in simulators indicate that the inspiratory pressure, the positive end expiratory pressure (PEEP), the tidal volume and the inspiratory time are administered more consistently using the T-piece resuscitator compared with the self-inflating bag. ${ }^{45}$ Despite these advantages regarding lung mechanics, three clinical trials in newborns demonstrate controversial results regarding short-term and long-term outcomes with the T-piece. ${ }^{6-8}$ In 2011, Dawson et $a l^{6}$ analysed 80 newborns $<29$ weeks, randomised to ventilation in the delivery room with the T-piece or the self-inflating bag, and found no differences regarding oxygen saturation at $5 \mathrm{~min}$ after birth, frequency of tracheal intubation and surfactant administration in the delivery room. The study was not designed to assess long-term outcomes, but its description showed no differences regarding bronchopulmonary dysplasia (BPD), grade IV intraventricular haemorrhage (IVH) and hospital mortality. In 2014, Szyld et $a l^{7}$ evaluated 195 VLBW neonates ventilated at birth: 85 with T-piece and 110 with self-inflating bag. The T-piece resuscitator decreased the need for intubation in the delivery room and the frequency of BPD. In 2015, Thakur et $a l^{8}$ studied 37 patients with gestational age $<34$ weeks, of whom 19 were ventilated in the delivery room with the T-piece resuscitator and 18 with the self-inflating bag. The use of the T-piece led to a shorter duration of positive pressure ventilation and a lower frequency of intubation at birth, with diminishing need for mechanical ventilation in the neonatal intensive care unit (NICU), although hospital mortality was similar between groups. Thus, given the lack of compelling data on benefits in the long term, in 2015, the International Liaison Committee on Resuscitation stated that there was insufficient evidence to recommend using the T-piece resuscitator instead of the self-inflating bag for resuscitation at birth and suggested the need of further research. ${ }^{5}$

The goal of this study was to determine whether the use of the T-piece resuscitator in VLBW infants who received positive pressure ventilation at birth, compared with the self-inflating bag, increased survival to hospital discharge without BPD, IVH grades III-IV and periventricular leucomalacia (PVL). In addition, as a secondary outcome, this study analysed whether the use of the T-piece, compared with the self-inflating bag, increased the chance of the newborn having a fifth-minute Apgar score of $7-10$.

\section{METHODS}

This is a pragmatic prospective cohort study with data obtained from the database registry of the Brazilian Network on Neonatal Research. Data from the 20 academic tertiary care public hospitals that participated in the network for the entire duration of the study period were analysed. All centres agreed to have their data included in the study, and the Research Ethics Committee of the coordinating institution approved the project without needing patients' informed consent.

All patients born between January 2014 and December 2015 with gestational age between $23^{0 / 7}$ and $33^{6 / 7}$ weeks, birth weight between 400 and $1499 \mathrm{~g}$, without malformations, who received positive pressure ventilation at birth were included in the study. Patients transferred to other institutions until 27 days after birth were excluded because we could not retrieve data about outcomes.
The 20 hospitals are high-risk pregnancy referral centres. Neonatal resuscitation teams followed the 2011 guidelines of the Neonatal Resuscitation Program of the Brazilian Society of Pediatrics, ${ }^{9}$ which introduced the possibility of using the T-piece resuscitator or the self-inflating bag as the first option for ventilation at birth for preterm neonates. In the present study, the decision to use the T-piece resuscitator and/or the self-inflating bag was at the discretion of each resuscitation team that assisted the patient at birth. The T-piece resuscitator was either the Neopuff (Fisher \& Paykel Healthcare, Auckland, New Zealand) or the Babypuff (Fanem Ltda, São Paulo, Brazil). All centres started the T-piece ventilation with a PEEP of $5 \mathrm{cmH}_{2} \mathrm{O}$. All self-inflating bags contained no PEEP valve. According to the 2011 Brazilian guidelines, the positive pressure ventilation, regardless of the equipment available, started with an oxygen concentration of $40 \%$ for newborns with gestational age less $<34$ weeks.

The patients who met the inclusion criteria were allocated into two groups: (1) T-piece, neonates ventilated at birth exclusively with a T-piece resuscitator with facial mask and/or endotracheal tube; and (2) self-inflating bag, infants who received any positive pressure ventilation at birth with a self-inflating bag by facial mask and/or endotracheal tube.

Gestational age, in completed weeks, was determined by the best obstetric estimate. Small for gestational age was defined according to Olsen et al. ${ }^{10}$ Hypothermia on admission to NICU was determined by an axillary temperature $<36.0^{\circ} \mathrm{C}^{11}$ The Score for Neonatal Acute Physiology and Perinatal Extension, Version II severity score was calculated within the first 12 hours of life. ${ }^{12}$ Patent ductus arteriosus was considered present when patients were submitted to pharmacological and/or surgical closure. Late-onset sepsis was defined by the presence of clinical signs after 72 hours of life with positive blood culture. Necrotising enterocolitis was considered in the presence of intestinal pneumatosis or pneumoperitoneum. BPD was defined when there was use of any inspired oxygen fraction above 0.21 in the corrected gestational age of 36 weeks. ${ }^{13}$ IVH was diagnosed via head ultrasound and classified according to Papile et al. ${ }^{14} \mathrm{PVL}$ was diagnosed when cystic lesions of intraparenchymal white matter were observed by head ultrasound during hospital stay. ${ }^{15}$

The primary outcome was survival to hospital discharge without BPD, IVH grades III-IV and PVL. The secondary outcome was the presence of a fifth-minute Apgar score of 7-10. Given the chance of the primary outcome in the study centres to be approximately $50 \%,{ }^{16}$ with a $10 \%$ difference between the studied groups and a power of $80 \%$, it was necessary to include at least 387 infants in each group.

The independent variables associated with the outcomes were defined by logistic regression. Since the variables associated with the fifth-minute Apgar score of 7-10 and the survival to hospital discharge without BPD, IVH grades III-IV and PVL vary between extreme preterm infants and very/moderate preterm infants, logistic models for the whole group of patients and for the neonates divided according to gestational age were built. The models included all maternal and neonatal variables with a $p$ value $<0.20$ on univariate analysis. The independent variables were removed one by one from the model according to their significance, adjusted by the Wald test. The fit of the model was evaluated by Hosmer-Lemeshow goodness-of-fit test. SPSS V.21.0 software was used.

\section{RESULTS}

In the 20 centres, 3021 neonates with gestational age of $23^{0 / 7-}$ $33^{6 / 7}$ weeks and birth weight of 400-1499 g were born without 


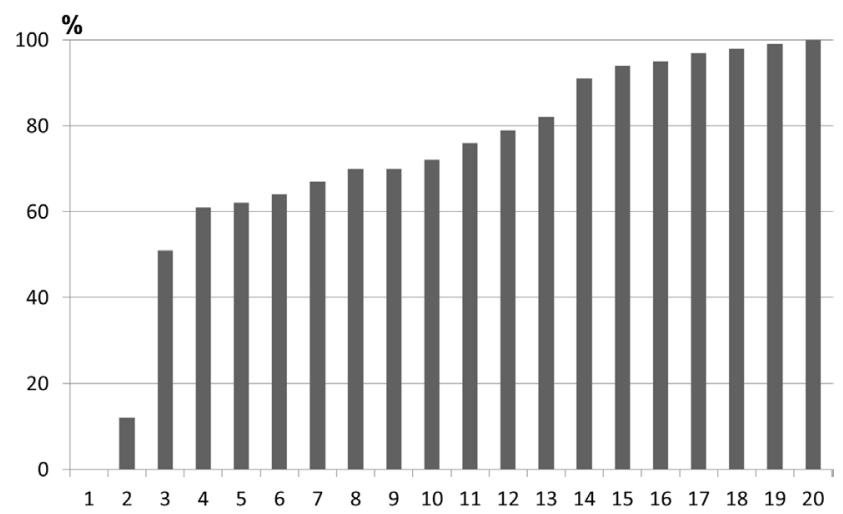

Figure 1 Frequency of T-piece use in the 1962 ventilated preterm neonates at birth, according to birth centre.

malformations in 2014-2015 and 2010 (67\%) received positive pressure ventilation at birth. Of these, 48 patients were transferred to other hospitals until 27 days after birth. Therefore, the study population was 1962 newborns who met the inclusion criteria. Among them, 1456 (74\%) were ventilated only with the T-piece, while $506(26 \%)$ received, at some point of resuscitation, ventilation with the self-inflating bag. The frequency of neonates ventilated only with the T-piece varied from $0 \%$ to $100 \%$ among centres (figure 1). The distribution of patients regarding the equipment for ventilation and the interface used (facial mask, endotracheal tube or both) is shown in figure 2 for the 1248 infants with a gestational age of 23-28 weeks, and in figure 3 for the 714 infants with 29-33 weeks. In relation to the use of oxygen, $836(57 \%)$ of those ventilated with the T-piece and $371(73 \%)$ of those ventilated with the self-inflating bag received $100 \%$ oxygen during resuscitation in the delivery room.

The maternal demographic characteristics of the 1962 neonates grouped according to the device used in ventilation in the delivery room are displayed in table 1 , and the neonatal characteristics and evaluated outcomes are shown in table 2 . The frequency of the main outcome of the study, that is, survival to hospital discharge without BPD, IVH (grades III/IV) or PVL, ranged among the studied units from $27 \%$ to $59 \%$ (9\%-40\% for the 23-28 weekers, and 56\%-88\% for the 29-33 weekers).

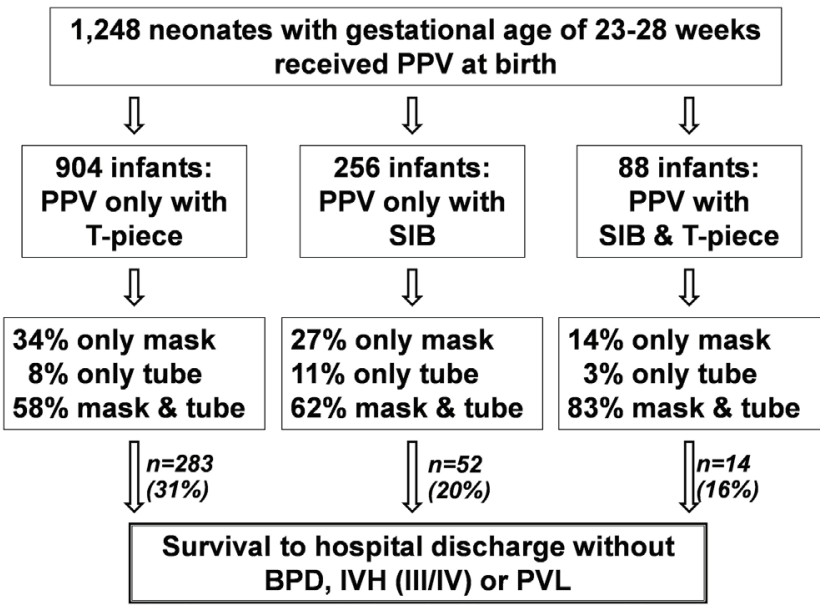

Figure 2 Distribution of the neonates with 23-28 weeks of gestational age according to the interface used for positive pressure ventilation at birth and main outcome. BPD, bronchopulmonary dysplasia; IVH, intraventricular haemorrhage; PPV, positive pressure ventilation; PVL, periventricular leucomalacia; SIB, self-inflating bag.

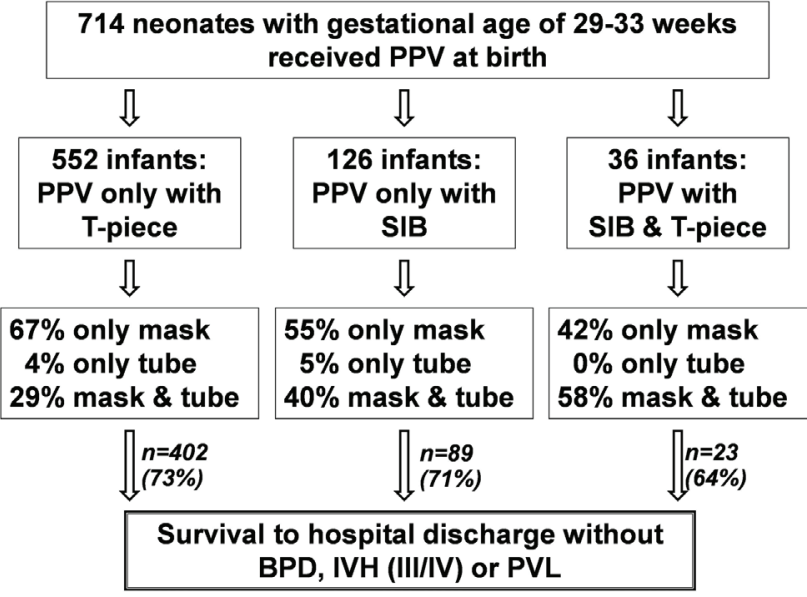

Figure 3 Distribution of the neonates with 29-33 weeks of gestational age according to the interface used for positive pressure ventilation at birth and main outcome. BPD, bronchopulmonary dysplasia; IVH, intraventricular haemorrhage; PPV, positive pressure ventilation; PVL, periventricular leucomalacia; SIB, self-inflating bag.

When the variables associated with the presence of a fifthminute Apgar score of 7-10 were analysed, the logistic regression adjusted for birth centre, maternal age, maternal schooling, prenatal care, maternal hypertension, multiple pregnancy and small for gestational age showed associations with the following variables, expressed in ORs (95\% CIs): antenatal steroid use: 1.43 (1.11 to 1.83 ), C-section 1.50 (1.18 to 1.91 ), chorioamnionitis 0.73 (0.54 to 0.99$)$, gestational age $<29$ weeks 0.40 (0.31 to 0.52 ), male newborn 0.77 (0.61 to 0.96 ), ventilation only with the T-piece at birth 1.95 (1.47 to 2.58$)$ and advanced resuscitation 0.10 (0.07 to 0.15$)$. This regression model has a good fit (Hosmer-Lemeshow goodness of fit: $p=0.877$ ). The variables associated with the presence of a fifth-minute Apgar score of 7-10 for the 1246 infants with 23-28 weeks and for the 714 neonates with 29-32 weeks of gestational age are shown in table 3 .

Logistic regression adjusted for birth centre, maternal characteristics and obstetric and neonatal morbidities showed that the T-piece resuscitator increased the chance of survival to hospital discharge without BPD, IVH (grades III/IV) or PVL for the 1962 neonates with 23-33 weeks of gestational age: OR 1.38 (95\% CI 1.06 to 1.80$)$; Hosmer-Lemeshow goodness of fit: 0.695 . The variables associated with the chance of survival to hospital discharge without BPD, IVH (grades III/IV) or PVL according to gestational age are shown in table 4.

\section{DISCUSSION}

The use of T-piece for positive pressure ventilation at birth of preterm neonates was associated with an increased chance of survival to hospital discharge without BPD, severe IVH or PVL, adjusted for possible confounding variables. It was also associated with an increased chance of a fifth-minute Apgar score of 7-10. According to the literature review, this study is the first with a significant number of patients that collected data from daily clinical assistance showing the benefits of using specific device for positive pressure ventilation in the delivery room in terms of relevant outcomes at hospital discharge.

It is interesting to note, first, the variability in the use of the T-piece between the various centres, and second the fast incorporation of the equipment in the delivery rooms of public Brazilian university hospitals. Figure 1 shows that, in 2014 and 2015, the T-piece was used for $90 \%$ or more of the preterm neonates who received ventilation at birth in seven centres, and 60\%-80\% of 


\section{Original article}

Table 1 Maternal characteristics of the 1962 infants classified according to positive pressure ventilation at birth with T-piece resuscitator or selfinflating bag

\begin{tabular}{lccc}
\hline & T-piece, $\mathbf{n = 1 4 5 6}$ & Self-inflating bag, $\mathbf{n = 5 0 6}$ & $\mathbf{p}$ Value \\
\hline Maternal age $<2$ years & $231(16 \%)$ & $101(20 \%)$ & 0.033 \\
\hline Schooling $<8$ years & $344(24 \%)$ & $140(28 \%)$ & 0.050 \\
Prenatal care & $1361(94 \%)$ & $458(91 \%)$ & 0.045 \\
Multiple gestation & $331(23 \%)$ & $112(22 \%)$ & 0.781 \\
\hline Hypertension & $546(38 \%)$ & $169(34 \%)$ & 0.147 \\
Diabetes & $103(7 \%)$ & $27(5 \%)$ & 0.198 \\
Chorioamnionitis & $200(14 \%)$ & $92(18 \%)$ & 0.015 \\
Abruption or previa & $152(11 \%)$ & $52(10 \%)$ & 0.920 \\
Antenatal steroid & $1114(77 \%)$ & $348(69 \%)$ & 0.001 \\
Caesarean section & $968(67 \%)$ & $286(57 \%)$ & $<0.001$ \\
Gestational age (weeks) & $28.2 \pm 2.5$ & $27.8 \pm 2.7$ & 0.005 \\
Gestational age $<29$ weeks & $904(62 \%)$ & $344(68 \%)$ & 0.018 \\
\hline
\end{tabular}

Abruption or previa: abruptio placentae or placenta previa.

the patients in ten centres. European studies indicate the use of the T-piece resuscitator in $31 \%$ of Irish maternity hospitals in $2008,{ }^{17} 45 \%$ of Spanish ones in $2009,{ }^{18} 41 \%$ of German units and $80 \%$ of Austrian ones in $2010 .{ }^{19}$ In UK, T-piece resuscitator was used by more than $90 \%$ of tertiary units and $78 \%$ of special care units in $2010 .^{20}$ The rapid adoption of this equipment by Brazilian university units indicates the embodiment of the 2011 neonatal resuscitation national guidelines, which suggested the use of the T-piece for neonates with gestational ages $<34$ weeks. ${ }^{9}$

Low birth weight neonates ventilated only with the T-piece, compared with those ventilated with the self-inflating bag at birth, was associated with a lower frequency of endotracheal intubation in the delivery room, surfactant administration and invasive mechanical ventilation in the NICU, as well as a lower length of hospital stay. These results differ from those presented by Dawson $e t a l^{6}$ in a randomised clinical trial with infants younger than 29 weeks. It is possible that the observational design of our study led to the enrolment of healthier infants who were non-urgently delivered in the T-piece group (tables 1 and 2), since the use of this equipment requires anticipation of its need. On the other hand, the study by Dawson et $a l^{6}$ had only 80 patients and it may not have had the sample

Table 2 Neonatal characteristics and outcomes of the 1962 infants classified according to positive pressure ventilation at birth with T-piece resuscitator or self-inflating bag

\begin{tabular}{|c|c|c|c|}
\hline & T-piece, $n=1456$ & Self-inflating bag, $n=506$ & $\mathrm{p}$ Value \\
\hline Birth weight $(\mathrm{g})$ & $969 \pm 277$ & $941 \pm 279$ & 0.968 \\
\hline Birth weight $<750 \mathrm{~g}$ & $374(26 \%)$ & $149(29 \%)$ & 0.099 \\
\hline Male & $745(51 \%)$ & $258(51 \%)$ & 0.945 \\
\hline Small for gestational age & $453(31 \%)$ & $147(29 \%)$ & 0.386 \\
\hline Endotracheal intubation in the delivery room & $782(54 \%)$ & $340(67 \%)$ & $<0.001$ \\
\hline CPAP in the delivery room & $763(53 \%)$ & $159(32 \%)$ & $<0.001$ \\
\hline Fifth-minute Apgar score of 7-10 & $1104(76 \%)$ & $322(64 \%)$ & $<0.001$ \\
\hline Hypothermia at NICU admission & $732(52 \%)$ & $310(67 \%)$ & $<0.001$ \\
\hline SNAPPE II $>40$ & $381(27 \%)$ & $168(36 \%)$ & $<0.001$ \\
\hline Respiratory distress syndrome & $1149(81 \%)$ & $401(83 \%)$ & 0.313 \\
\hline Air leaks & $99(7 \%)$ & $28(6 \%)$ & 0.369 \\
\hline Pulmonary hypertension & $120(9 \%)$ & $51(11 \%)$ & 0.163 \\
\hline Surfactant administration & $976(68 \%)$ & $377(77 \%)$ & $<0.001$ \\
\hline Mechanical ventilation by tracheal tube & $1094(77 \%)$ & $422(88 \%)$ & $<0.001$ \\
\hline Mechanical ventilation by tracheal tube (days) ${ }^{*}$ & $4(1-13)$ & $5(2-18)$ & $<0.001$ \\
\hline PDA with pharmacological or surgical closure & $350(25 \%)$ & $121(25 \%)$ & 0.866 \\
\hline Late-onset sepsis with positive blood culture & $375(27 \%)$ & $135(29 \%)$ & 0.371 \\
\hline Necrotising enterocolitis & $110(8 \%)$ & $42(9 \%)$ & 0.509 \\
\hline IVH grades III/IV & $145 / 1218(12 \%)$ & $60 / 376(16 \%)$ & 0.040 \\
\hline PVL & $95 / 1209(8 \%)$ & $46 / 373(12 \%)$ & 0.008 \\
\hline Death in the delivery room & $43(3 \%)$ & $25(5 \%)$ & 0035 \\
\hline Alive at hospital discharge & $1000(69 \%)$ & $282(56 \%)$ & $<0.001$ \\
\hline Survival to hospital discharge without BPD & 765 (53\%) & $198(39 \%)$ & $<0.001$ \\
\hline Survival without BPD, IVH (III/IV) or PVL & $685(47 \%)$ & $178(35 \%)$ & $<0.001$ \\
\hline
\end{tabular}

${ }^{*}$ Data displayed in median (percentile 25-75).

BPD, bronchopulmonary dysplasia; CPAP, continuous positive airway pressure; IVH, intraventricular haemorrhage (\% of those with head ultrasound); NICU, neonatal intensive care unit; PDA, patent ductus arteriosus; PVL, periventricular leucomalacia (\% of those with head ultrasound); SNAPPE II, Score for Neonatal Acute Physiology and Perinatal Extension, Version II. 
Table 3 Variables associated with a fifth-minute Apgar score of 7-10 according to gestational age

\begin{tabular}{lll}
\hline & OR & $95 \% \mathrm{Cl}$ \\
\hline Infants with 23-28 weeks of gestational age* & & \\
\hline Maternal schooling & 0.72 & 0.53 to 0.96 \\
\hline Chorioamnionitis & 0.70 & 0.50 to 0.98 \\
\hline Antenatal steroids & 1.57 & 1.17 to 2.10 \\
\hline Caesarean section & 1.61 & 1.23 to 2.11 \\
\hline Male sex & 0.68 & 0.52 to 0.89 \\
\hline Birth weight $<750 \mathrm{~g}$ & 0.55 & 0.42 to 0.72 \\
\hline Ventilation at birth with the T-piece & 1.52 & 1.14 to 2.03 \\
\hline Advanced resuscitation at birth & 0.10 & 0.06 to 0.16 \\
\hline Infants with 29-33 weeks of gestational age** & & \\
\hline Maternal hypertension & 1.84 & 1.16 to 2.92 \\
\hline Ventilation at birth with the T-piece & 2.43 & 1.50 to 3.90 \\
\hline Advanced resuscitation at birth & 0.10 & 0.04 to 0.25 \\
\hline
\end{tabular}

* Logistic regression model adjusted for birth centre, prenatal care, maternal hypertension and multiple gestation (Hosmer-Lemeshow: $p=0.463$ ).

**Logistic regression model adjusted for birth centre, maternal race, and birth weight $<1000 \mathrm{~g}$ (Hosmer-Lemeshow: $\mathrm{p}=0.995$ ).

†Advanced resuscitation at birth: ventilation via endotracheal tube + chest compressions and/or medication.

Table 4 Variables associated with survival to hospital discharge without bronchopulmonary dysplasia, intraventricular haemorrhage grades III/IV and periventricular leucomalacia, according to gestational age

\begin{tabular}{lll}
\hline & OR & $95 \% \mathrm{Cl}$ \\
\hline Infants with 23-28 weeks of gestational age & & \\
\hline Caesarean section & 1.49 & 1.10 to 2.02 \\
\hline Birth weight $<750 \mathrm{~g}$ & 0.13 & 0.09 to 0.19 \\
\hline Male & 0.64 & 0.47 to 0.86 \\
\hline Ventilation at birth with the T-piece & 1.76 & 1.24 to 2.50 \\
\hline Advanced resuscitation & 0.44 & 0.22 to 0.89 \\
\hline Fifth-minute Apgar score of 7-10 & 1.48 & 1.04 to 2.11 \\
\hline Respiratory distress syndrome & 0.42 & 0.25 to 0.69 \\
\hline Air leaks & 0.34 & 0.17 to 0.68 \\
\hline Pulmonary hypertension & 0.280 & 0.15 to 0.52 \\
\hline Late-onset sepsis with positive blood culture & 0.58 & 0.42 to 0.81 \\
\hline Infants with 28-33 weeks of gestational age** & & \\
\hline Maternal hypertension & 0.16 & 0.06 to 0.41 \\
\hline Caesarean section & 1.69 & 1.08 to 1.67 \\
\hline Birth weight $<1000 \mathrm{~g}$ & 0.22 & 0.13 to 0.38 \\
\hline Advanced resuscitation & 0.31 & 0.11 to 0.91 \\
\hline Respiratory distress syndrome & 0.41 & 0.26 to 0.65 \\
\hline Air leaks & 0.16 & 0.04 to 0.69 \\
\hline PDA with pharmacological and/or surgical & 0.48 & 0.27 to 0.84 \\
treatment & & \\
\hline Late-onset sepsis with positive blood culture & 0.44 & 0.28 to 0.68 \\
\hline Necrotising enterocolitis & 0.44 & 0.20 to 0.97 \\
\hline L & & \\
\hline
\end{tabular}

*Logistic regression model adjusted for birth centre, maternal age and race, antenatal steroids, hypothermia on intensive care admission, PDA with pharmacological and/or surgical treatment, and necrotising enterocolitis (HosmerLemeshow: $p=0.987$ )

** Logistic regression model adjusted for birth centre, maternal race, prenatal care, multiple gestation, previa/abruption, multiple gestation, small for gestational age, fifth-minute Apgar score of 7-10 and ventilation at birth with T-piece (HosmerLemeshow: $p=0.329$ ).

PDA, persistent ductus arteriosus. power necessary to find differences between groups ventilated with different devices. In agreement with data presented here, two randomised clinical trials show that the use of the T-piece in preterm infants, compared with the self-inflating bag, decreased the need for intubation in the delivery room and mechanical ventilation in NICU. ${ }^{78}$ To address the possible bias of an observational study, we assessed the short-term outcome adjusted for variables that would indicate a better vitality of patients who received T-piece ventilation at birth. After adjusting for maternal morbidity, antenatal steroid use, delivery mode, gestational age, sex of the newborn and first minute Apgar score, ventilation with the T-piece at birth was associated with an increased chance of fifth-minute Apgar score of $7-10$ by $37 \%$, suggesting that this device provides more effective ventilation for resuscitation in the delivery room. Animal and human data indicate that providing PEEP in a critical period for maintenance of functional residual capacity is important to remove fluid from the airways. ${ }^{21}$ In addition, the inspiratory pressure during the ventilation with self-inflating bags is highly variable and the expiratory pressure is inconsistent, which might be harmful during the resuscitation at birth of preterm infants. ${ }^{67}$

Regarding outcomes at hospital discharge, positive pressure ventilation with the T-piece in the delivery room was associated with a higher chance of survival without BPD, severe IVH or PVL by 38\%, adjusted for maternal and neonatal characteristics and birth centre. The hospital mortality rate of $32 \%$ in this preterm cohort of Brazilian infants is three times higher than those reported by North American neonatal networks and population studies in Switzerland and Japan, which ranged from $9 \%$ to $13 \%$ for VLBW preterm neonates born between 2008 and 2012. ${ }^{22-24}$ South American data from 2001 to 2011, with 8234 VLBW infants, are similar to the Brazilian data, with $26 \%$ hospital deaths. ${ }^{25}$ When considering only the survivors, the frequency of morbidities evaluated in this study was higher than those described for North American centres from the Vermont-Oxford Network: BPD $25 \%$ vs $26 \%$, severe IVH $8 \%$ vs $6 \%$ and PVL $9 \%$ vs $3 \% .^{22}$ The use of a brief intervention in the delivery room, that is, positive pressure ventilation with T-piece, was associated with an increase in survival to hospital discharge without major morbidities. By decreasing the need for endotracheal intubation and increasing continuous positive airway pressure with facial mask soon after birth, the use of T-piece in the delivery room has favoured less invasive mechanical ventilation during the infant's hospital stay. Invasive mechanical ventilation implies the availability of adequate infrastructure in terms of equipment and human resources in neonatal units. Although Brazilian university units have adequate physical structure and equipment for the care of high-risk neonates, the presence of registered nurses for NICU beds is lower than that reported in developed countries. ${ }^{26}$ It is known that there is a direct relationship between the proportion of nurses and neonatal hospital outcome. ${ }^{27}$ Thus, the use of T-piece for ventilation at birth may constitute an intervention with favourable impact on survival to hospital discharge without major morbidities in centres with neonatal care conditions similar to those described here.

The main limitation of this investigation is its observational design. As a strength, this is a pragmatic trial that assesses the effectiveness of the T-tube in a real-world setting with the use of a large database analysed in a manner intended to adequately adjust for potential confounders. ${ }^{28}$ It is interesting to note that the analysed cohort corresponds to $4.5 \%$ of VLBW neonates born each year in Brazil, ${ }^{29}$ with data collected in a prospective and systematic way 
and with strict definitions of each event and continuous input in an electronic database, whose consistency is verified by an independent committee.

This study is the first that highlights the effectiveness of T-piece resuscitator ventilation in improving relevant outcomes in preterm neonates. The use of the T-piece in the delivery room implies the availability of resuscitation teams with professionals able to anticipate the need for positive pressure ventilation at birth and to prepare equipment for immediate use.

\section{Author affiliations}

'Division of Neonatal Medicine, Federal University of São Paulo, São Paulo, Brazil ${ }^{2}$ Department of Pediatrics, Faculdade de Medicina de Ribeirão Preto, University of São Paulo, Ribeirão Preto, Brazil

${ }^{3}$ Department of Pediatrics, Universidade Federal do Maranhão, São Luis, Brazil ${ }^{4}$ Department of Pediatrics, Faculdade de Ciências Médicas da Universidade Estadual de Campinas, Campinas, Brazil

${ }^{5}$ Department of Pediatrics, Faculdade de Medicina da Universidade de São Paulo, São Paulo, Brazil

${ }^{6}$ Division of Neonatology, Faculdade de Medicinade Botucatu da Universidade Estadual Paulista, Botucatu, Brazil

${ }^{7}$ Division of Neonatology, Fundacao Oswaldo Cruz, Rio de Janeiro, Brazil

${ }^{8}$ Department of Pediatrics, Hospital São Lucas - Faculdade de Medicina da Pontifícia Universidade Católica do Rio Grande do Sul, Porto Alegre, Brazil

${ }^{9}$ Division of Neonatology, Universidade Federal do Rio Grande do Sul/Hospital de Clínicas de Porto Alegre, Porto Alegre, Brazil

${ }^{10}$ Department of Pediatrics, Hospital Universitário Pedro Ernesto - Universidade do Estado de Rio de Janeiro, Rio de Janeiro, Brazil

${ }^{11}$ Division of Neonatology, Universidade Federal de Minas Gerais, Belo Horizonte, Brazil

${ }^{12}$ Pediatrics, Universidade Federal de Uberlandia, Uberlandia, Brazil

${ }^{13}$ Department of Pediatrics, Maternidade Hilda Brandão - Faculdade de Ciências

Médicas de Minas Gerais, Belo Horizonte, Brazil

${ }^{14}$ Department of Pediatrics, University of São Paulo, School of Medicine, SP - Brasil, São Paulo, Brazil

${ }^{15}$ Neonatal Division, Hospital Estadual Sumaré, Sumaré, Brazil

${ }^{16}$ Neonatal Unit, Hospital Geral de Pirajussara, Taboão da Serra, Brazil

${ }^{17}$ Neonatal Unit, Hospital Estadual de Diadema, Diadema, Brazil

${ }^{18}$ Department of Pediatrics, Hospital Universitário - Universidade Estadual de Londrina, Curitiba, Brazil

${ }^{19}$ Department of Pediatrics, Hospital de Clínicas - Universidade Federal do Paraná, Curitiba, Brazil

${ }^{20}$ Department of Pediatrics, Instituto de Medicina Integral Professor Fernando Figueira, Recife, Brazil

${ }^{21}$ Department of Pediatrics, Instituto Fernandes Figueira - FIOCRUZ, Rio de Janeiro, Brazil

Correction notice This paper has been amended since it was published Online First. Owing to a scripting error, some of the publisher names in the references were replaced with 'BMJ Publishing Group'. This only affected the full text version, not the PDF. We have since corrected these errors and the correct publishers have been inserted into the references.

Contributors RG and MFBdA have participated in the concept and design, analysis and interpretation of data, and drafting and revising the manuscript. All other authors have participated in the design of the study, collection and interpretation of data and revising the manuscript. All authors listed on the manuscript approved the submission of this version of the manuscript and take full responsibility for the manuscript.

\section{Competing interests None declared.}

Patient consent This is a prospective collection of data of all very low birth weight neonates born in 20 Brazilian public hospitals and affiliated to the Brazilian Network on Neonatal Research. There are no interventions and the present study used this database. The IRB approved the use of this registry for the present study.

Ethics approval Committee on Ethics in Research of Instituto Fernandes Figueira Fundação Oswaldo Cruz, Rio de Janeiro, Brazil.

Provenance and peer review Not commissioned; externally peer reviewed.

Data sharing statement There are no additional unpublished data from the submitted study. The database is available, upon request, from the main authors (RG and MFBdA).

(C) Article author(s) (or their employer(s) unless otherwise stated in the text of the article) 2018. All rights reserved. No commercial use is permitted unless otherwise expressly granted.

\section{REFERENCES}

1 Rede Brasileira de Pesquisas Neonatais. http://www.redeneon atal.fiocruz.br/. (accessed 19 Aug 2016).

2 Stoll BJ, Hansen NI, Bell EF, et al. Neonatal outcomes of extremely preterm infants from the NICHD Neonatal Research Network. Pediatrics 2010;126:443-56

3 Hooper SB, Te Pas AB, Kitchen MJ. Respiratory transition in the newborn: a threephase process. Arch Dis Child Fetal Neonatal Ed 2016;101:F266-71.

4 Hawkes CP, Ryan CA, Dempsey EM. Comparison of the T-piece resuscitator with other neonatal manual ventilation devices: a qualitative review. Resuscitation 2012;83:797-802.

5 Perlman JM, Wyllie J, Kattwinkel J, et al. Part 7: Neonatal Resuscitation: 2015 International Consensus on Cardiopulmonary Resuscitation and Emergency Cardiovascular Care Science With Treatment Recommendations. Circulation 2015;132:S204-41.

6 Dawson JA, Schmölzer GM, Kamlin CO, et al. Oxygenation with T-piece versus self-inflating bag for ventilation of extremely preterm infants at birth: a randomized controlled trial. J Pediatr 2011;158:912-8.

7 Szyld E, Aguilar A, Musante GA, et al. Comparison of devices for newborn ventilation in the delivery room. J Pediatr 2014;165:234-9.

8 Thakur A, Saluja S, Modi M, et al. T-piece or self inflating bag for positive pressure ventilation during delivery room resuscitation: an RCT. Resuscitation 2015;90:21-4.

9 Guinsburg R, Ameida MFB, Anchieta LM, eds. Curso de Reanimação do Prematuro na Sala de Parto: manual Didático do Instrutor. Rio de Janeiro: Sociedade Brasileira de Pediatria, 2012.

10 Olsen IE, Groveman SA, Lawson ML, et al. New intrauterine growth curves based on United States data. Pediatrics 2010;125:e214-24.

11 World Health Organization. Thermal protection of the Newborn: a Practical Guide Geneva:WHO, 1997.

12 Richardson DK, Corcoran JD, Escobar GJ, et al. SNAP-II and SNAPPE-II: simplified newborn illness severity and mortality risk scores. J Pediatr 2001:138:92-100

13 Jobe AH, Bancalari E. Bronchopulmonary dysplasia. Am J Respir Crit Care Med 2001;163:1723-9.

14 Papile LA, Burstein J, Burstein R, et al. Incidence and evolution of subependymal and intraventricular hemorrhage: a study of infants with birth weights less than $1,500 \mathrm{gm}$. J Pediatr 1978:92:529-34.

15 de Vries LS, Eken P, Dubowitz LM. The spectrum of leukomalacia using cranial ultrasound. Behav Brain Res 1992:49:1-6.

16 Guinsburg R, de Almeida MF, de Castro JS, et al. Death or survival with major morbidity in VLBW infants born at Brazilian neonatal research network centers. J Matern Fetal Neonatal Med 2016;29:1005-9.

17 Braima 0, Ryan CA. Neonatal resuscitation program quidelines 2006: ready, steady, can't go!. Ir Med J 2008;101:142-4.

18 Iriondo M, Thió $\mathrm{M}$, Burón $\mathrm{E}$, et al. A survey of neonatal resuscitation in Spain: gaps between guidelines and practice. Acta Paediatr 2009;98:786-91.

19 Roehr CC, Gröbe S, Rüdiger M, et al. Delivery room management of very low birth weight infants in Germany, Austria and Switzerland--a comparison of protocols. Eur J Med Res 2010;15:493-503.

20 Mann C, Ward C, Grubb M, et al. Marked variation in newborn resuscitation practice: a national survey in the UK. Resuscitation 2012;83:607-11.

21 Hooper SB, Siew ML, Kitchen MJ, et al. Establishing functional residual capacity in the non-breathing infant. Semin Fetal Neonatal Med 2013;18:336-43.

22 Horbar JD, Carpenter JH, Badger GJ, et al. Mortality and neonatal morbidity among infants 501 to 1500 grams from 2000 to 2009. Pediatrics 2012;129:1019-26.

23 Rüegger C, Hegglin M, Adams M, et al. Population based trends in mortality, morbidity and treatment for very preterm- and very low birth weight infants over 12 years. BMC Pediatr 2012;12:17.

24 Kusuda S, Fujimura M, Uchiyama A, et al. Trends in morbidity and mortality among very-low-birth-weight infants from 2003 to 2008 in Japan. Pediatr Res 2012;72:531-8.

25 Fernández R, D'Apremont I, Domínguez A, et al. Survival and morbidity of very low birth weight infant in a South American neonatal network. Arch Argent Pediatr 2014:112:405-12.

26 Azevedo Bittencourt SD, Costa Reis LG, Ramos MM, et al. Structure in Brazilian maternity hospitals: key characteristics for quality of obstetric and neonatal care. Cad Saude Publica 2014;51-12.

27 Watson SI, Arulampalam W, Petrou S, et al. The effects of a one-to-one nurseto-patient ratio on the mortality rate in neonatal intensive care: a retrospective, longitudinal, population-based study. Arch Dis Child Fetal Neonatal Ed 2016;101:F195-200

28 Tyson J, Pedroza C, Wootton SH. Analyses of Large Data Bases and Pragmatic Clinical Trials: Advancing Comparative Effectiveness Research in a Learning Health Care System. Am J Perinatol 2015:32:1095-7.

29 Saúde BMda. Portal Da Saúde (homepage on the internet) Datasus: Estatísticas Vitais. 2017 http://www2.datasus.gov.br/DATASUS/index.php?area=0205 